\title{
Increasing negative emotions by reappraisal enhances subsequent cognitive control: A combined behavioral and electrophysiological study
}

\author{
Jason S. Moser, Steven B. Most, and Robert F. Simons \\ University of Delaware, Newark, Delaware
}

\begin{abstract}
To what degree do cognitively based strategies of emotion regulation impact subsequent cognitive control? Here, we investigated this question by interleaving a cognitive task with emotion regulation trials, where regulation occurred through cognitive reappraisal. In addition to obtaining self-reports of emotion regulation, we used the late positive potential (LPP) of the event-related brain potential as an objective index of emotion regulation. On each trial, participants maintained, decreased, or increased their emotional response to an unpleasant picture and then responded to a Stroop stimulus. Results revealed that (1) the magnitude of the LPP was decreased with reappraisal instructions to decrease negative emotion and were enhanced with reappraisal instructions to increase negative emotion; (2) after cognitive reappraisal was used to increase the intensity of negative emotion, RT interference in the subsequent Stroop trial was significantly reduced; and (3) increasing negative emotions by reappraisal also modulated the cognitive control-related sustained potential. These results suggest that increasing negative emotions by cognitive reappraisal heightens cognitive control, which may be sustained for a short time after the regulation event.
\end{abstract}

The ability to regulate one's own emotions is central to psychological and physical well-being (see Gross, 2007). Although several studies have demonstrated the widespread changes (experiential, physiological, etc.) that accompany the active regulation of emotion (for reviews, see Gross \& Thompson, 2007; Ochsner \& Gross, 2005), fewer have spoken directly to how such emotion regulation impacts the processing of subsequent or concurrent tasks. As Zelazo and Cunningham (2007) have noted, "one strives to regulate one's emotion (e.g., up-regulation or down-regulation) in order to foster the fulfillment of some other goal about which one cares" (p. 147). Therefore, it is important to examine what consequences emotion regulation has for subsequent cognitive control. In the present study, we ask, Does regulating emotion drain or prime the cognitive control resources necessary for subsequent attentionally demanding tasks?

There is reason to suggest that the type of regulation one employs largely determines whether cognitive resources are drained or primed. Researchers agree that not all emotion regulation strategies are equal. For example, people can rely on either behavioral or cognitive strategies to regulate their emotions (Gross \& Thompson, 2007). Behavioral emotion regulation involves the manipulation of outward expressions of emotion, either to suppress or to enhance the intensity of expression. Cognitive reappraisal, on the other hand, involves changing the way that one thinks about an emotion-evoking event in order to increase or decrease one's affective response.

Nearly all studies showing that emotion regulation drains cognitive resources have involved behavioral emotion regulation. The first clues about the cognitive effects of behavioral emotion regulation came from studies showing impaired memory for emotional films and pictures presented under behavioral suppression instructions (Richards \& Gross, 1999, 2000, 2006). Similarly, Bonanno, Papa, Lalande, Westphal, and Coifman (2004) showed impaired memory performance for emotional pictures presented during behavioral suppression and exaggeration. Most relevant to the present investigation, at least three studies have shown that behavioral emotion regulation drained available resources for subsequent cognitive processing. Schmeichel (2007) found that participants who exaggerated their outward, behavioral emotional expressions to an unpleasant film subsequently exhibited significantly reduced working memory span. Similarly, Shamosh and Gray (2007) reported that suppressing outward, behavioral emotional responses to an unpleasant film increased subsequent cognitive interference effects on a Stroop-like task. Finally, Inzlicht and Gutsell (2007) found that when participants suppressed their outward, behavioral emotional reactions to a sad movie, subsequent Stroop interference not only increased, but also was mediated by reduced action monitoring, as indexed by an 
event-related brain potential (ERP) associated with conflict and error detection (the error-related negativity, or ERN; Gehring, Goss, Coles, Meyer, \& Donchin, 1993). These findings are consistent with the notion that use of self-regulatory control - in this case, behavioral emotion regulation-draws on limited cognitive resources, thus leaving fewer resources for additional processing (see Muraven \& Baumeister, 2000). They are also consistent with the suggestion that behavioral emotion regulation involves internal monitoring of emotive behaviors and, therefore, leads to a withdrawal of resources from external stimuli (Richards \& Gross, 2000).

Only one study showed that cognitive reappraisal drains cognitive resources. Deveney and Pizzagalli (2008) investigated the impact of cognitively decreasing and increasing emotional responses to unpleasant pictures on subsequent emotional word processing. Although they failed to show any effects of reappraisal on subsequent behavioral performance in the emotional word identification task, cognitively increasing negative emotions led to subsequently reduced attention allocation to emotional words, as indexed by the P300 of the visually evoked ERP (Donchin, 1981), suggesting that negative affect usurps available cognitive resources (Ellis \& Ashbrook, 1988; Kliegel, Horn, \& Zimmer, 2003). Importantly, however, unlike all other studies of emotion regulation's effects on subsequent processing, this study examined the effects of cognitive emotion regulation on cognitive resources allocated toward subsequent emotional stimuli. Thus, this effect might speak more to how emotion regulation affects the availability of subsequent resources for emotionalnot nonemotional-processing.

Notably, some studies have shown that emotion regulation primes cognitive resources, and all of these have involved cognitive reappraisal; however, there are few such studies, and none have examined cognitive reappraisal's effects on cognitive control per se. Richards and Gross (2000) and Richards, Butler, and Gross (2003) found enhanced memory for pictures and emotional conversations under reappraisal instructions. Similarly, Dillon, Ritchey, Johnson, and LaBar (2007) showed that unpleasant pictures presented under reappraisal instructions to decrease and increase negative emotions were better remembered during a surprise recall test, with the strongest memory benefit shown for increasing negative emotions. The enhanced memory for information presented under reappraisal instructions has been interpreted as reflecting increased attention to and elaboration on the emotioneliciting stimuli (Dillon et al., 2007). Although such effects are not directly related to the impact of reappraisal on subsequent cognitive control, it is possible that by increasing attentional engagement, the effects of reappraisal carry over and enhance control over responses to stimuli in close temporal proximity.

With the present study, then, our aim was to build on previous findings by specifically examining the effects of cognitive reappraisal on subsequent cognitive control. Participants maintained, decreased, and increased emotional responses to unpleasant pictures via cognitive reappraisal instructions and subsequently performed a nu- merical version of the Stroop task (e.g., Windes, 1968), in which they responded to arrays of one, two, or three digits by indicating the number of items in the array. In each array, all the digits had the same identity, which could be " 1 ," "2," or " 3 ." In this task, attentional conflict arises when the number of digits (i.e., the task-relevant dimension) is incongruent with the identity of the digits (i.e., the task-irrelevant dimension). For example, the array " 333 " would be congruent, but the array " 222 " would be incongruent. Thus, participants must exercise selective attention and respond to one stimulus dimension while ignoring the other; in general, incongruent trials elicit more errors and longer response times (RTs) than do congruent trials. We chose the Stroop task because of its likely overlap with emotion regulation in terms of supporting cognitive functions and neural structures. Specifically, both Stroop performance and emotion regulation rely on cognitive control instantiated by a frontoparietal network (Egner, Delano, \& Hirsch, 2007; Liston, Matalon, Hare, Davidson, \& Casey, 2006; McClure, Botvinick, Yeung, Greene, \& Cohen, 2007; E. K. Miller \& Cohen, 2001; Ochsner \& Gross, 2005, 2007). Together, the anterior cingulate cortex (ACC), prefrontal cortex (PFC), and parietal cortex coordinate the detection and resolution of conflicts between response and stimulus representations and implement behavioral and cognitive adjustments, which facilitate both successful Stroop performance and regulation of emotion. To the extent that cognitive reappraisal and Stroop performance recruit an overlapping cognitive control mechanism, one might predict a change in Stroop performance subsequent to this strategy of emotion regulation. Specifically, we tested whether the engagement of such overlapping control mechanisms in the service of emotion regulation carries over to a temporally proximal cognitive control task, as would be reflected by improved Stroop performance. We also examined modulations of Strooprelated ERP components following emotion regulation. Specifically, we examined modulations of two cognitive control-related ERPs: the N450 and sustained potential (SP). These components have been associated with cognitive control processes in numerous Stroop tasks and show sources in frontal cortices, including the ACC and PFC (Lansbergen, van Hell, \& Kenemans, 2007; West, 2003; West, Jakubek, Wymbs, Perry, \& Moore, 2005).

In addition to examining the impact of reappraisal instructions on subsequent behavioral Stroop performance, and because self-report measures of emotional experience can be influenced by demand characteristics, we built on evidence that a late positive potential (LPP) of the visually evoked ERP can serve as an objective, neurophysiological index of emotional regulation. The LPP is a centroparietally maximal ERP component peaking approximately $300 \mathrm{msec}$ post-stimulus-onset and lasting up to several seconds, which is particularly notable for being commensurate with ratings of emotional arousal: It exhibits its largest amplitudes in response to highly arousing unpleasant and pleasant stimuli (for a review, see Olofsson, Nordin, Sequeira, \& Polich, 2008). Most important for the purposes of the present experiment, the LPP has proven sensitive to reappraisal instructions in several previous 
experiments (Hajcak \& Nieuwenhuis, 2006; Krompinger, Moser, \& Simons, 2008; Moser, Hajcak, Bukay, \& Simons, 2006; Moser, Krompinger, Dietz, \& Simons, 2009). In general, the amplitude of the LPP decreases when participants follow reappraisal instructions to decrease emotions elicited by either unpleasant or pleasant pictures (Hajcak \& Nieuwenhuis, 2006; Krompinger et al., 2008; Moser et al., 2006; Moser et al., 2009) and increases when participants follow reappraisal instructions to increase emotions elicited by unpleasant pictures (Moser et al., 2009).

Although the primary reason for measuring the LPP was to provide an objective index of the impact of reappraisal processes during emotion regulation, another reason for examining the LPP was its ostensible relationship to cognitive processes, including attention and memory elicited by emotional stimuli (Olofsson et al., 2008), as well as cognitive control (Larson, Kaufman, \& Perlstein, 2009). ERPs in the time window of the LPP have been linked to a phasic locus coeruleus (LC)-dependent norepinephrine (NE) response aimed at facilitating appropriate action to task-relevant stimuli. The LC-NE hypothesis suggests that NE enhances sensory gain in cortical regions involved in selective attention and response generation (Nieuwenhuis, Aston-Jones, \& Cohen, 2005). Thus, to the extent that the LPP reflects cognitive control processes during reappraisal, we anticipated that LPP modulation would be correlated with subsequent Stroop performance.

Thus, the present study had two primary aims: (1) to examine the effects of reappraisal on subsequent cognitive control, as indexed by Stroop performance, and (2) to replicate previous findings that reappraisal instructions modulate the LPP. Although our primary measure of Strooprelated cognitive control was behavioral performance, we also explored Stroop-related ERP modulations. We explored the degree to which Stroop-related performance and ERPs are modulated following reappraisal instructions to decrease and increase one's emotional response, with the prediction that reappraisal would lead to subsequently enhanced cognitive performance.

\section{METHOD}

\section{Participants}

Twenty-nine undergraduate students (21 of them female) participated in the present study for course credit. To encourage the participants to follow task instructions closely, they were told that the two most successful emotion regulators, as measured by brain activity, would be awarded $\$ 20$ in bonus money. At the completion of the study, individual ERP averages were calculated for each participant, and the two students who evidenced the largest emotion regulation effects on ERP measures were awarded the bonus. Two participants were excluded from analyses because of poor numerical Stroop performance (i.e., performance $<50 \%$ correct on one or more Stroop trial types).

\section{Stimuli and Procedures}

The stimulus set comprised 50 unpleasant, high-arousing color images taken from the International Affective Picture System (IAPS; Lang, Bradley, \& Cuthbert, 1999). ${ }^{1}$ The unpleasant picture set included images of mutilation and threat (human and animal). Mean normative valence and arousal ratings for the pictures were 2.55 and 6.48 , respectively. In addition, numerical Stroop stimuli consisted of the numbers 1, 2, and 3 arranged one, two, or three times in a horizontal row on each trial. Thus, there were instances of congruent (e.g., "22”) and incongruent (e.g., "222") Stroop stimuli.

After the participants had received a general description of the experiment, electroencephalogram/electrooculogram (EEG/EOG) sensor electrodes were attached. The participants were seated approximately $0.5 \mathrm{~m}$ directly in front of a computer monitor and were given detailed task instructions. The participants performed two blocks of the emotion regulation picture-viewing task, administered on a 17-in. monitor via a Celeron D class computer using Presentation software (Neurobehavioral Systems, Inc.) to control the presentation and timing of all the stimuli. The emotion regulation pictureviewing paradigm was adapted from Jackson, Malmstadt, Larson, and Davidson (2000) and Ochsner et al. (2004) for use with ERPs.

On each trial, the participants first heard, over computer speakers, a spoken instruction word - maintain, decrease, or increaseindicating that their task during the viewing of the upcoming picture was to view and respond naturally to the content of the upcoming photo, to decrease their emotional response to the content of the photo, or to increase their emotional response to the content of the photo, respectively. During the auditory presentation of the instruction word, a gray fixation cross was presented at the center of the screen in order to orient the participants' attention to the upcoming picture. The duration of the cue word and fixation presentation was $2 \mathrm{sec}$. An IAPS picture was then displayed for $2 \mathrm{sec}$ and occupied the entire monitor screen. Following a random interval that ranged from 800 to $1,500 \mathrm{msec}$ after the offset of the IAPS picture, the numerical Stroop stimulus appeared for $500 \mathrm{msec}$. The participants were instructed to use one of three keys to indicate as quickly and accurately as possible the number of digits presented on the screen. The period between the offset of the Stroop stimulus and the onset of the next cue word lasted $3 \mathrm{sec}$, during which time the participants were instructed to relax. Each block contained 100 trials, with one block including 50 unpleasant pictures paired once with a maintain and once with a decrease instruction; the other block included the same 50 unpleasant pictures paired once with a maintain and once with an increase instruction. Thus, the participants viewed each unpleasant picture four times. Increase and decrease trials were not mixed within block, in order to avoid potential task switching effects (e.g., switching from decreasing to increasing on successive trials; see Monsell, 2003) that might obscure differences between regulation and passive-viewing conditions. For each instruction, within each block, half of the Stroop stimuli were congruent, and the other half were incongruent. The order of the two blocks was counterbalanced across participants, and within each block, the order of trials contributing to that block's 2 (instruction) $\times 2$ (Stroop congruence) design was randomized.

Prior to each of the experimental blocks, the participants performed two practice blocks in order to familiarize themselves with the task and emotion regulation instructions (described in more detail below). During the first practice block, the participants were given detailed instructions regarding the order and timing of the stimuli, the meaning of each cue to be presented, and how to perform the Stroop task. The participants then engaged in approximately 5 trials in which they were asked to verbally generate proper appraisals of the stimuli in accordance with the instructional cue and to respond quickly and accurately to the Stroop stimuli. This practice block allowed the experimenter to help shape the participants' appraisals and determine that the participants understood the Stroop task. During the second practice block, the participants performed 10 trials of the task and were asked to continue generating appropriate appraisals in accordance with the instructional cue, but silently, as they would be doing during the experimental blocks. Finally, the participants were again reminded of the task procedures and emotion regulation instructions and were given a last chance to ask any additional questions.

Following the last experimental block, the physiological sensors were removed, and the participants completed a manipulation check questionnaire in which they were asked to report the strategies that 
they had used for maintain, decrease, and increase trials. All the participants reported using the instructed techniques to maintain, decrease, and increase their emotional reactions to the pictures. The participants also reported on the strength of emotion experienced $(1=$ very weak; 7 = very strong $)$ and the degree of effort utilized $(1=$ very little $;=$ very $m u c h)$ on maintain, decrease, and increase trials. One participant did not complete the posttask questionnaires, and thus analyses involving it are based on 26 participants. Ratings were taken at the conclusion of the experiment, and not during it, primarily because such trial-by-trial ratings would have likely disrupted the assessment of the effects of emotion regulation on subsequent performance of the Stroop task. Moreover, we felt that including trial-by-trail affect and effort ratings would have further taxed the participants' resources and added another layer of complexity to the experiment.

\section{Emotion Regulation Instructions}

Instructions for the emotion regulation picture-viewing task were adapted from Ochsner et al. (2004). For maintain trials, the participants were instructed simply to view the pictures and respond naturally to the content. The participants were explicitly instructed not to alter their natural responses in any way and to maintain eye gaze on the computer screen for all trial types, to reduce the potential confounding effects of significant eye movements. For the decrease and increase trials, the participants were given two strategies that corresponded to Ochsner et al.'s self- and situation-focused strategies. For the decrease trials, the participants were given the option to view the picture from a detached, third-person perspective, as would someone with no personal attachment to the pictured events or as if it were fake/from a movie (self-focused), or to imagine that the scenario in the pictured scene had a positive outcome (situation focused). The participants were explicitly told not to generate unrelated positive thoughts or images to decrease their responses. ${ }^{2}$ For the increase trials, the participants were given the option to view the picture from an attached or first-person perspective, as would someone personally invested or personally partaking in the pictured events (self-focused), or to imagine that the scenario in the pictured scene worsened (situation focused). The participants were explicitly told not to generate unrelated negative thoughts or images to increase their responses. ${ }^{3}$

\section{Psychophysiological Recording, Data Reduction, and Analysis}

The EEG was recorded using an ECI electrocap. Recordings were taken from four locations along the midline: frontal $(\mathrm{Fz})$, frontocentral $(\mathrm{FCz})$, central $(\mathrm{Cz})$, and parietal $(\mathrm{Pz})$. In addition, Med Associates tin electrodes were placed on the left and right mastoids (M1 and M2, respectively). During the recording, all activity was referenced to $\mathrm{Cz}$. The EOG generated from blinks and vertical eye movements was also recorded using Med Associates miniature electrodes placed approximately $1 \mathrm{~cm}$ above and below the participant's right eye. The right earlobe served as a ground site. All EEG/EOG electrode impedances were below $10 \mathrm{~K} \Omega$, and the data from all channels were recorded by a Grass Model 7D polygraph with Grass Model 7P1G preamplifiers (band-pass $=0.008-35 \mathrm{~Hz}$ ).

All bioelectric signals were digitized on a laboratory microcomputer using VPM software (Cook, 1999). The EEG was sampled at $200 \mathrm{~Hz}$. For LPP analysis, data collection began $500 \mathrm{msec}$ prior to instruction cue onset and continued for $4,500 \mathrm{msec}$, until the offset of the picture. For analysis of Stroop-related ERPs, data collection began $200 \mathrm{msec}$ prior to onset of the Stroop stimulus and continued for 1,000 msec. Offline, the EEG for each trial was corrected for vertical EOG artifacts according to previously developed methods (Gratton, Coles, \& Donchin, 1983; G. A. Miller, Gratton, $\&$ Yee, 1988) and then was rereferenced to the average activity of the mastoid electrodes. Trials were rejected and not counted in the subsequent analysis if there was an excessive physiological artifact (i.e., $25 \mathrm{msec}$ of invariant analog data on any channel or $\mathrm{A} / \mathrm{D}$ values on any channel that equaled that converter's minimum or maxi- mum value; $M$ artifact-free trials for maintain trials in the decrease block $=43.67, M$ for decrease trials $=43.30, M$ for maintain trials in the increase block $=43.93, M$ for increase trials $=43.07$ ). For both behavioral and ERP analyses, Stroop error trials and trials on which the RT was shorter than $200 \mathrm{msec}$ or longer than $2,000 \mathrm{msec}$ were discarded. Thus, Stroop- and emotion-regulation-related ERPs and performance data were derived from the same set of trials. Singletrial EEG data were low-pass filtered at $20 \mathrm{~Hz}$ with a 51-weight FIR digital filter, as per Cook and Miller (1992).

For the LPP analyses, ERPs were constructed by separately averaging maintain and decrease trials from the decrease block and maintain and increase trials from the increase block at each recording site. For each ERP, the average activity in the 0 - to 100 -msec window prior to picture onset served as the baseline. The LPP was then measured as the average activity in the 300 - to 1,800 -msec post-picture-onset window, to capture the maximal and sustained LPP activity.

For Stroop-locked ERP analyses, ERPs were constructed by separately averaging across trials in each cell of a 2 (congruent vs. incongruent trial) $\times 2$ (maintain vs. regulate instruction) $\times$ 2 (decrease block vs. increase block) matrix. Activity in the 0 - to $100-\mathrm{msec}$ window prior to Stroop onset served as the baseline for $\mathrm{N} 450$ and SP analyses. The N450 was then scored as the peak activity in the 300- to 500-msec post-Stroop-onset window, and the SP was measured as the peak activity in the 750 - to 900 -msec postStroop-onset window.

Behavioral and ERP data were statistically evaluated using SPSS (Version 15.0) general linear model software with GreenhouseGeisser correction applied to $p$ values associated with multiple $d f$ repeated measures comparisons where appropriate. The effect of regulation instruction block order (decrease first vs. increase first) was evaluated for the behavioral and LPP findings and was not significant in either case $(p s>.15)$ and, thus, is not further reported on below. Further analyses proceeded as follows. (1) First, posttask emotional strength and effort ratings were submitted to a one-way repeated measures rANOVA with the factor of regulation instruction (maintain vs. decrease vs. increase) to confirm appropriate emotion modulation by regulation instruction and to examine how effortful the participants found it to decrease and increase negative emotion. (2) Next, LPP data were analyzed for each block separately to confirm electrophysiological modulations by decrease and increase instructions, as compared with their respective maintain instructions. To rule out possible baseline differences between the maintain conditions in the decrease versus the increase block, a 2 (block: decrease vs. increase) $\times 4$ (site: $\mathrm{Fz}, \mathrm{FCz}, \mathrm{Cz}$, or $\mathrm{Pz}$ ) rANOVA was conducted on the maintain conditions in each block and revealed no effects of block ( $p$ s $>.31)$. LPP magnitudes from each block were then submitted to separate 2 (regulation instruction: maintain vs. regulate) $\times 4$ (site: $\mathrm{Fz}, \mathrm{FCz}, \mathrm{Cz}$, or Pz) rANOVAs. (3) Last, behavioral and Stroop-locked ERP data were analyzed separately by block to investigate the Stroop-related RT and ERP modulations by decrease and increase instructions, relative to their respective maintain instructions. The behavioral performance measures were submitted to a 2 (regulation instruction: maintain vs. regulate) $\times$ 2 (Stroop congruence: congruent vs. incongruent) rANOVA. N450 and SP magnitudes were submitted to separate 2 (regulation instruction: maintain vs. regulate) $\times 2$ (Stroop congruence: congruent vs. incongruent) $\times 4$ (site: $\mathrm{Fz}, \mathrm{FCz}, \mathrm{Cz}$, or Pz) rANOVAs.

\section{RESULTS}

\section{Posttask Questionnaire Findings}

A review of the posttask emotion regulation strategies questionnaire revealed that the participants reported following the instructions closely and were quite uniform in the strategy utilized for decrease and increase trials. Specifically, 20 of the 26 (76.9\%) participants who completed the posttask questionnaire reported using the self- 
focused strategy to decrease emotions (e.g., "I detached myself. I would look at the image as something not real"), and 23 of the $26(88.5 \%)$ participants reported using the self-focused strategy to increase negative emotions (e.g., "I pictured the people in the picture as me or someone in my family"). Thus, the majority of the participants reported using the self-focused strategy for both decrease and increase trials.

The ANOVA conducted on the emotional strength ratings confirmed significant modulation of emotional experience across the three trial types $[F(2,50)=46.55$, $p<.001]$, such that decrease trials were associated with the weakest emotional experience $(M=2.71, S D=$ $0.18)$, maintain trials were associated with moderately strong unpleasant reactions $(M=4.21, S D=0.21)$, and increase trials were associated with the strongest emotional reactions $(M=5.50, S D=0.25$; all comparisons, $p \leq .001)$. Analysis of the effort ratings showed a significant effect of regulation instruction $[F(2,52)=6.30, p=$ $.004]$, such that maintain trials required the least amount of effort $(M=3.11, S D=1.85)$, as compared with decrease $(M=4.54, S D=1.78 ; p=.003)$ and increase $(M=4.74, S D=1.72 ; p=.004)$ trials. The participants reported that decrease and increase trials required equal effort $(p=.706)$.

\section{ERP and Behavioral Modulations by Decrease Instructions}

Stimulus-locked grand-averaged ERPs at $\mathrm{Fz}, \mathrm{FCz}, \mathrm{Cz}$, and Pz depicting the LPP for the decrease block are presented in Figure 1. An analysis of the entire LPP in the decrease block revealed a significant site effect $[F(3,78)=$ $8.46, p=.001]$, replicating previous reports that the LPP was largest at the parietal electrode (for a review, see Olofsson et al., 2008). Although the main effect of regulation instruction was not significant $(F<1)$, the site $\times$ regulation instruction interaction did reach significance $[F(3,78)=3.20, p=.037]$. Consistent with our previous work (Moser et al., 2006; Moser et al., 2009), follow-up tests at each electrode revealed that decrease was asso-

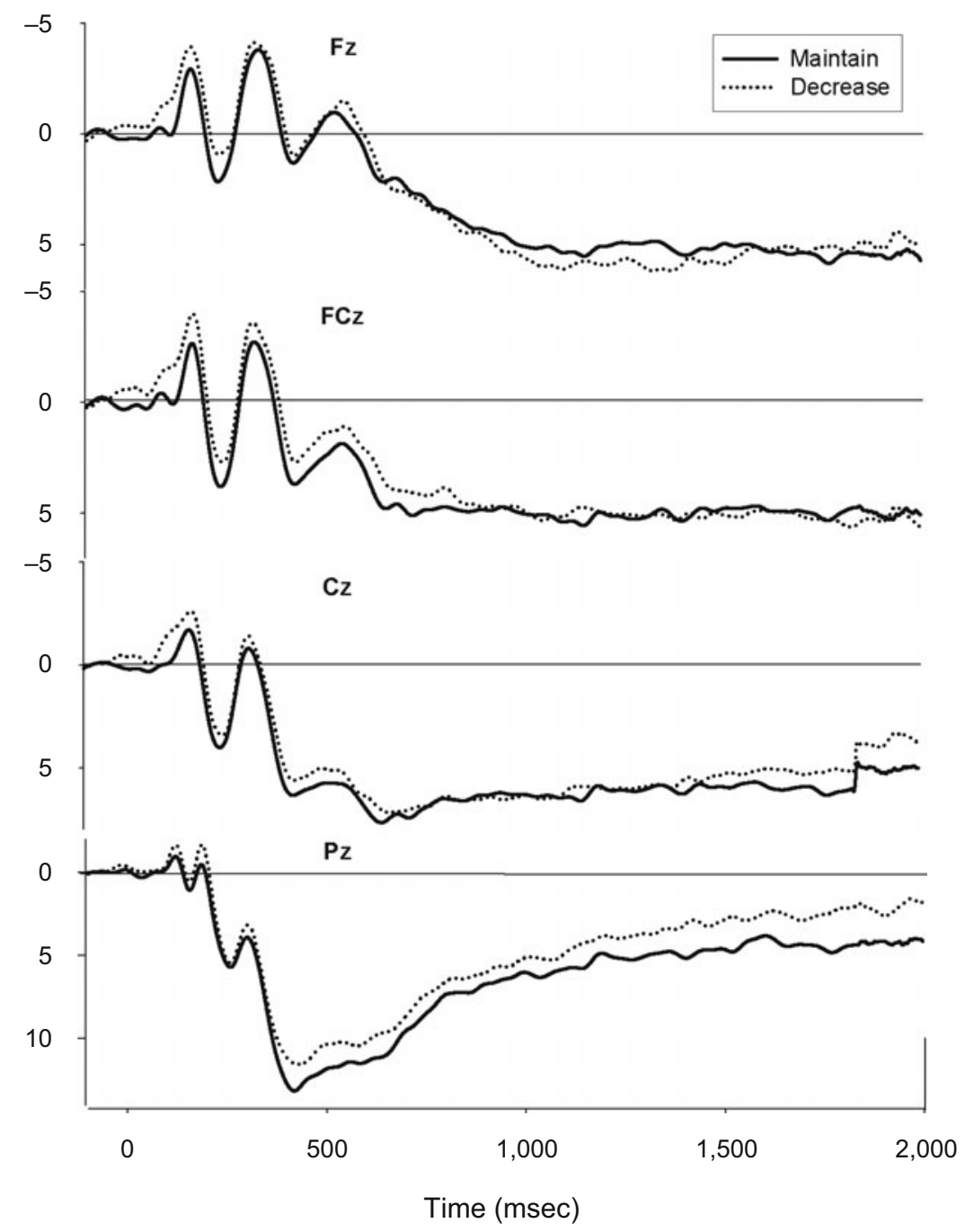

Figure 1. Picture-locked ERPs at frontal (Fz), frontocentral (FCz), central $(\mathrm{Cz})$, and parietal $(\mathrm{Pz})$ locations for the decrease block. Time point 0 represents picture onset. 
Table 1

Means (With Standard Deviations)

for Latent Positive Potential Measures $(\mu \mathrm{V})$

\begin{tabular}{|c|c|c|c|c|c|c|c|c|}
\hline \multirow[b]{3}{*}{ Location } & \multicolumn{4}{|c|}{ Decrease Block } & \multicolumn{4}{|c|}{ Increase Block } \\
\hline & \multicolumn{2}{|c|}{ Maintain } & \multicolumn{2}{|c|}{ Decrease } & \multicolumn{2}{|c|}{ Maintain } & \multicolumn{2}{|c|}{ Increase } \\
\hline & $M$ & $S D$ & $M$ & $S D$ & $M$ & $S D$ & $M$ & $S D$ \\
\hline $\mathrm{Fz}$ & 3.50 & 3.48 & 3.69 & 4.59 & 3.40 & 3.61 & 4.86 & 3.96 \\
\hline $\mathrm{FCz}$ & 4.23 & 4.81 & 3.89 & 5.83 & 4.47 & 3.44 & 5.82 & 3.94 \\
\hline $\mathrm{Cz}$ & 5.96 & 4.39 & 5.58 & 4.54 & 5.51 & 3.67 & 6.71 & 4.39 \\
\hline $\mathrm{Pz}$ & 6.96 & 4.25 & 5.67 & 4.20 & 6.59 & 3.66 & 7.50 & 4.07 \\
\hline
\end{tabular}

Note-LPP, 300-1,800 msec. Fz, frontal; FCz, frontocentral; Cz, central; Pz, parietal.

ciated with a smaller LPP than was maintain at the $\mathrm{Pz}$ electrode only $[t(26)=2.62, p=.014$; all other $t \mathrm{~s}<1$; see Table 1]. Thus, the LPP results confirmed the effectiveness, at a neurophysiological level, of reappraisal processes in the decrease block.

Follow-up analysis of the LPP in shorter successive time windows in the decrease block revealed only a significant site effect in the 350- to $650-\mathrm{msec}$ time window $[F(3,78)=124.59, p<.001$; all other $p \mathrm{~s}>.15]$, showing that the early LPP was largest at the parietal electrode. In the 650- to $1,000-\mathrm{msec}$ time window, a similar significant site effect emerged $[F(3,78)=10.21, p<.001$; all other $p \mathrm{~s}>.29]$. In the late LPP time window, $1,000-1,800 \mathrm{msec}$, there was a significant regulation instruction $\times$ site interaction $[F(3,78)=5.97, p=.004]$, such that the LPP elicited on decrease trials $(M=3.61 \mu \mathrm{V}, S D=4.34)$ was smaller than that on maintain trials $(M=4.98 \mu \mathrm{V}, S D=$ $4.16)$ at the parietal lead only $[t(26)=2.33, p=.028$; all other $p \mathrm{~s}>.48]$. Neither the main effect of regulation instruction nor the main effect of site reached significance during this time window ( $p \mathrm{~s}>.18$ ).

An analysis of the RT data in the decrease block revealed only a significant effect of Stroop congruence $[F(1,26)=$ $117.83, p<.001$; all other $F \mathrm{~s}<1]$, with incongruent stimuli eliciting longer RTs than did congruent stimuli. An analysis of the percentage correct data also showed only a significant effect of Stroop congruence $[F(1,26)=11.38$, $p=.002$; all other $p \mathrm{~s}>.29$ ], with poorer performance on incongruent than on congruent trials (see Table 2).

A 2 (regulation instruction) $\times 2$ (Stroop congruence) $\times$ 4 (site) rANOVA was conducted on the peak amplitude of the N450 elicited by Stroop trials. Only the site effect was significant in the decrease block, indicating a frontal distribution $[F(3,78)=17.86, p<.001$; all other $p$ s $>$ .17; see Table 3]. Similarly, an analysis of the peak SP showed only a significant site effect, indicating a frontal distribution $[F(3,78)=30.26, p<.001$; all other $p$ s $>$ .26; see Table 4].

In sum, although the participants showed evidence for engagement of reappraisal processes aimed at decreasing negative emotion, as evidenced by smaller LPPs on decrease trials, cognitively decreasing negative emotion had no effect on subsequent Stroop performance or the Stroop-locked N450 and SP.

\section{ERP and Behavioral Modulations by Increase Instructions}

Stimulus-locked grand-averaged ERPs at $\mathrm{Fz}, \mathrm{FCz}, \mathrm{Cz}$, and $\mathrm{Pz}$ depicting the LPP for the increase block are presented in Figure 2. An analysis of the entire LPP in the increase block revealed a significant site effect $[F(3,78)=$ $13.12, p<.001]$, showing that the LPP was largest at the parietal electrode. The main effect of regulation instruction was also significant $[F(1,26)=4.46, p=.045]$, indicating larger LPP magnitude on increase trials than on maintain trials (see Table 1). The interaction between site and regulation instruction was not significant $(F<1)$. Thus, the LPP results confirmed the effectiveness, at a neurophysiological level, of reappraisal processes in the increase block.

A follow-up analysis of the LPP in shorter successive time windows in the increase block revealed only a significant site effect in the 350- to 650-msec time window $[F(3,78)=113.78, p<.001]$, showing that the early LPP was largest at the parietal electrode. The regulation instruction effect was marginal $[F(1,26)=3.50, p=.073]$. The regulation instruction $\times$ site interaction did not approach significance $(F<1)$. In the 650 - to 1,000 -msec time window, the regulation instruction effect emerged $[F(1,26)=8.83, p=.006]$, indicating larger LPPs on increase $(M=7.00 \mu \mathrm{V}, S D=3.88)$ than on maintain $(M=$ $5.33 \mu \mathrm{V}, S D=3.29$ ) trials. The site effect also continued to

Table 2

Means (With Standard Deviations) for Behavioral Performance Measures

\begin{tabular}{|c|c|c|c|c|c|c|c|c|}
\hline \multirow[b]{3}{*}{ Measure } & \multicolumn{4}{|c|}{ Decrease Block } & \multicolumn{4}{|c|}{ Increase Block } \\
\hline & \multicolumn{2}{|c|}{ Maintain } & \multicolumn{2}{|c|}{ Decrease } & \multicolumn{2}{|c|}{ Maintain } & \multicolumn{2}{|c|}{ Increase } \\
\hline & $M$ & $S D$ & $M$ & $S D$ & $M$ & $S D$ & $M$ & $S D$ \\
\hline Congruent response time & 599.45 & 93.19 & 591.45 & 98.06 & 585.87 & 89.88 & 591.85 & 102.82 \\
\hline Incongruent response time & 649.16 & 93.56 & 651.05 & 95.81 & 653.11 & 101.09 & 636.03 & 94.41 \\
\hline Congruent $\%$ correct & 95.70 & 7.99 & 95.56 & 9.24 & 91.70 & 7.44 & 93.19 & 5.18 \\
\hline Incongruent $\%$ correct & 91.56 & 10.01 & 93.33 & 10.11 & 91.70 & 8.80 & 92.30 & 10.22 \\
\hline
\end{tabular}


Table 3

Means (With Standard Deviations) for N450 $(\mu \mathrm{V})$

\begin{tabular}{|c|c|c|c|c|c|c|c|c|c|c|c|c|c|c|c|c|}
\hline \multirow{3}{*}{ Location } & \multicolumn{8}{|c|}{ Decrease Block } & \multicolumn{8}{|c|}{ Increase Block } \\
\hline & \multicolumn{4}{|c|}{ Maintain } & \multicolumn{4}{|c|}{ Decrease } & \multicolumn{4}{|c|}{ Maintain } & \multicolumn{4}{|c|}{ Increase } \\
\hline & \multicolumn{2}{|c|}{ Congruent } & \multicolumn{2}{|c|}{ Incongruent } & \multicolumn{2}{|c|}{ Congruent } & \multicolumn{2}{|c|}{ Incongruent } & \multicolumn{2}{|c|}{ Congruent } & \multicolumn{2}{|c|}{ Incongruent } & \multicolumn{2}{|c|}{ Congruent } & \multicolumn{2}{|c|}{ Incongruent } \\
\hline $\mathrm{Fz}$ & -5.71 & 9.27 & -6.47 & 8.40 & -6.24 & 9.22 & -6.69 & 11.12 & -5.71 & 10.20 & -6.55 & 7.52 & -5.67 & 7.80 & -7.95 & 11.27 \\
\hline $\mathrm{FCz}$ & -4.67 & 8.05 & -5.59 & 7.82 & -4.43 & 7.71 & -5.35 & 9.13 & -4.84 & 8.47 & -5.61 & 6.41 & -5.28 & 7.85 & -7.50 & 9.36 \\
\hline $\mathrm{Cz}$ & -3.26 & 6.75 & -3.96 & 6.13 & -3.50 & 7.12 & -3.95 & 7.08 & -3.63 & 5.95 & -4.29 & 4.94 & -3.71 & 5.23 & -5.58 & 6.63 \\
\hline
\end{tabular}

Note-Fz, frontal; FCz, frontocentral; $\mathrm{Cz}$, central; Pz, parietal.

show a parietal distribution of the $\operatorname{LPP}[F(3,78)=13.55$, $p<.001]$. The regulation instruction $\times$ site interaction did not approach significance $(F<1)$. In the late LPP time window, $1,000-1,800 \mathrm{msec}$, there was a marginal effect of regulation instruction $[F(1,26)=4.02, p=.055]$, showing somewhat larger LPPs on increase than on maintain trials. Neither the main effect of site nor its interaction with regulation instruction reached significance during this time window ( $p$ s $>.17)$.

An analysis of the RT data in the increase block revealed both a significant effect of Stroop congruence $[F(1,26)=108.89, p<.001]$ and a significant regulation instruction $\times$ Stroop congruence interaction $[F(1,26)=$ $7.55, p=.011]$, with Stroop interference (incongruent $\mathrm{RT}$ - congruent RT) significantly reduced in the increase condition $(M=44.18, S D=36.27)$, as compared with the maintain condition $(M=67.25, S D=34.44)$. Followup analyses revealed that this effect was driven by a significant change in incongruent RT between increase $(M=$ 636.03, $S D=94.41)$ and maintain $(M=653.11, S D=$ 101.09) $[t(26)=2.80, p=.01]$ trials, and not by a change in congruent RT ( $t<1$; see Table 2 and Figure 3). Finally, an analysis of the percentage correct data in the increase block showed no significant effects (all $p \mathrm{~s}>.22$ ).

Stimulus-locked grand-averaged ERPs at $\mathrm{Fz}, \mathrm{FCz}, \mathrm{Cz}$, and Pz depicting the N450 and SP for congruent and incongruent trials on increase trials are presented in Figure 4 . The 2 (regulation instruction) $\times 2$ (Stroop congruence) $\times 4$ (site) rANOVA conducted on the peak amplitude of the N450 showed a main effect of Stroop congruence $[F(1,26)=9.73, p=.004]$, indicating greater negativity for incongruent stimuli (see Table 3). A significant site effect also emerged $[F(3,78)=11.34, p=.001]$, indicating greater negativity at frontal sites (see Table 3 ). None of the other effects were significant $(p \mathrm{~s}>.13)$.
The 2 (regulation instruction) $\times 2$ (Stroop congruence) $\times 4$ (site) rANOVA conducted on the peak amplitude of the SP in the increase block revealed a significant regulation instruction $\times$ Stroop congruence interaction $[F(1,26)=6.65, p=.016]$. An examination of the effect of Stroop congruence in each condition revealed no significant effect of Stroop congruence in the maintain condition $[F(1,26)=1.05, p=.32]$. The effect of Stroop congruence was significant, however, in the increase condition $[F(1,26)=6.19, p=.02]$, showing larger SP amplitude to incongruent than to congruent Stroop trials (see Table 4). Last, the main effect of site for the omnibus rANOVA was also significant $[F(3,78)=41.63, p<.001]$, showing a frontal distribution of the SP (see Table 4).

We then calculated difference scores for the Stroop interference effect (incongruent - congruent) reflected in RT and SP and entered them into a correlation analysis. The RT interference effect was significantly correlated with the effect of congruence on SP [measured at $\mathrm{Cz}$; $r(27)=-.44, p=.02]$, indicating that as the difference in SP increased, the RT interference effect decreased.

Last, we calculated the LPP modulation difference score by increase instructions (increase - maintain) in the windows where it was significant - that is, in the entire window and the 650- to 1,000-msec time windowand correlated it with the Stroop RT and SP interference effects. None of these correlations were significant $(r \mathrm{~s}<$ $.26, p \mathrm{~s}>.18$ ).

In summary, the participants showed neurophysiological markers of reappraisal aimed at increasing negative emotion, as evidenced by larger LPPs on increase trials. Importantly, the subsequent Stroop RT interference effect was reduced and the Stroop-locked SP interference effect was enhanced on increase trials. The difference in SP elicited by incongruent versus congruent Stroop trials

Table 4

Means (With Standard Deviations) for the Sustained Potential $(\mu \mathrm{V})$

\begin{tabular}{|c|c|c|c|c|c|c|c|c|c|c|c|c|c|c|c|c|}
\hline \multirow[b]{4}{*}{ Location } & \multicolumn{8}{|c|}{ Decrease Block } & \multicolumn{8}{|c|}{ Increase Block } \\
\hline & \multicolumn{4}{|c|}{ Maintain } & \multicolumn{4}{|c|}{ Decrease } & \multicolumn{4}{|c|}{ Maintain } & \multicolumn{4}{|c|}{ Increase } \\
\hline & \multicolumn{2}{|c|}{ Congruent } & \multicolumn{2}{|c|}{ Incongruent } & \multicolumn{2}{|c|}{ Congruent } & \multicolumn{2}{|c|}{ Incongruent } & \multicolumn{2}{|c|}{ Congruent } & \multicolumn{2}{|c|}{ Incongruent } & \multicolumn{2}{|c|}{ Congruent } & \multicolumn{2}{|c|}{ Incongruent } \\
\hline & $M$ & $S D$ & $M$ & $S D$ & $M$ & $S D$ & $M$ & $S D$ & $M$ & $S D$ & $M$ & $S D$ & $M$ & $S D$ & $M$ & $S D$ \\
\hline $\mathrm{Fz}$ & 16.54 & 19.04 & 19.20 & 17.42 & 18.15 & 13.56 & 17.72 & 18.73 & 19.75 & 16.07 & 18.18 & 12.59 & 18.02 & 14.14 & 21.46 & 17.37 \\
\hline $\mathrm{FCz}$ & 11.09 & 13.79 & 13.62 & 12.72 & 13.56 & 12.24 & 12.56 & 13.83 & 13.80 & 11.50 & 12.59 & 10.68 & 11.01 & 3.94 & 13.70 & 13.64 \\
\hline $\mathrm{Cz}$ & 7.23 & 10.45 & 9.31 & 9.66 & 8.61 & 8.89 & 8.68 & 9.53 & 9.23 & 8.61 & 8.42 & 7.78 & 7.19 & 8.19 & 9.12 & 9.50 \\
\hline $\mathrm{Pz}$ & 2.96 & 6.47 & 4.29 & 6.47 & 3.34 & 6.25 & 3.89 & 5.93 & 4.43 & 5.99 & 4.02 & 5.63 & 2.56 & 5.25 & 4.23 & 5.45 \\
\hline
\end{tabular}

Note-Fz, frontal; FCz, frontocentral; $\mathrm{Cz}$, central; Pz, parietal. 


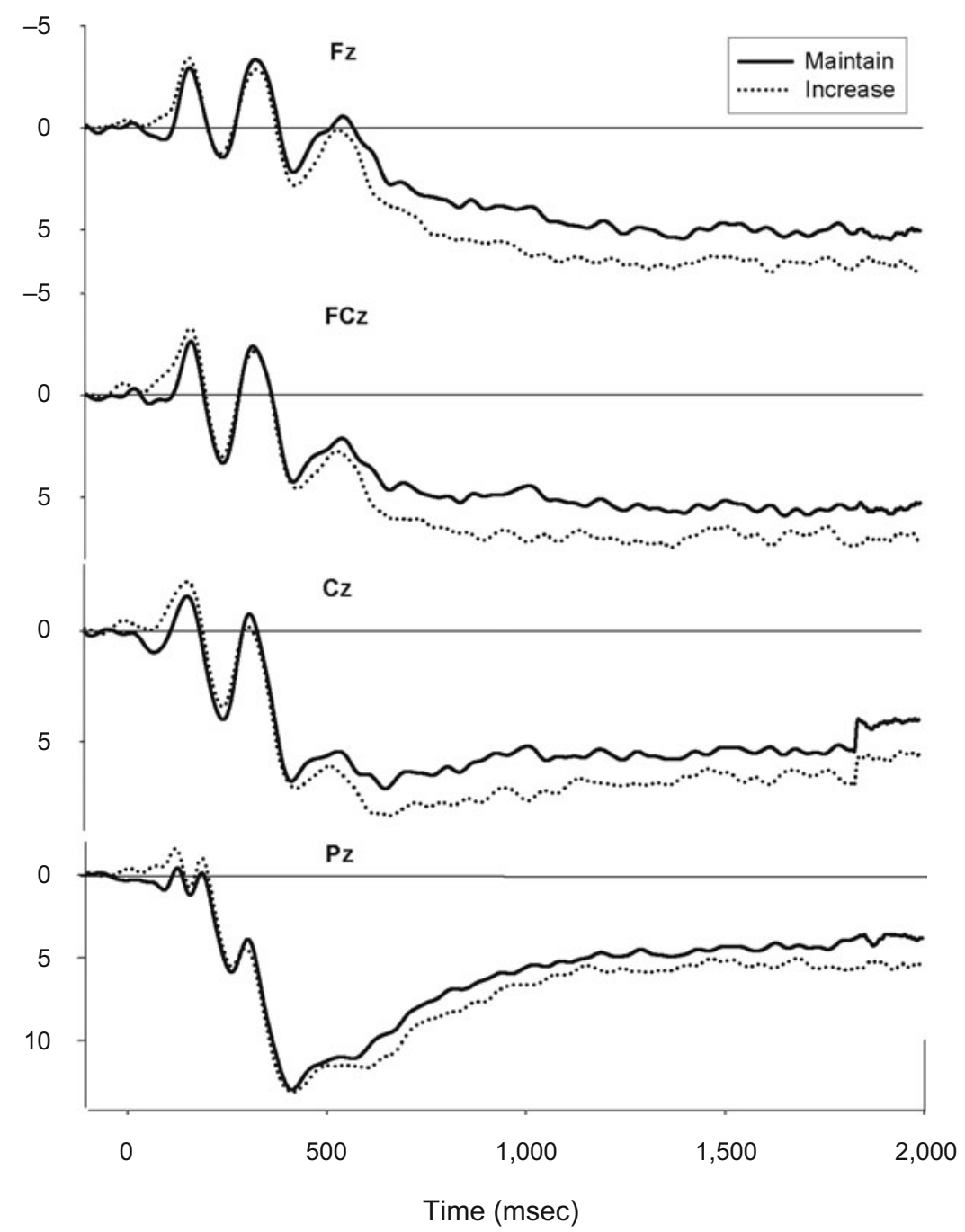

Figure 2. Picture-locked ERPs at frontal (Fz), frontocentral (FCz), central $(\mathrm{Cz})$, and parietal $(\mathrm{Pz})$ locations for the increase block. Time point 0 represents picture onset.

predicted the magnitude of the Stroop RT interference effect on increase trials, such that the larger the difference in sustained positivity, the smaller the RT interference effect. LPP modulation by increase instructions, however, did not significantly relate to RT or SP interference effects.

\section{DISCUSSION}

In the present study, we examined the effects of cognitive emotion regulation (reappraisal) on subsequent cognitive control. To ensure that the participants were engaged in reappraisal processes during the regulation instruction period, we measured modulations of the LPP in addition to self-report. As in our previous work (Moser et al., 2006; Moser et al., 2009), the LPP was significantly decreased under instructions to decrease negative emotion and was significantly enhanced under instructions to increase negative emotion. Following instructions to increase negative emotion, Stroop RT interference was significantly reduced. Moreover, the conflict-related SP was of greater amplitude on incongruent than on congruent trials following increase instructions, and this ERP modulation was negatively related to Stroop RT interference. Together, these findings suggest that increased cognitive engagement during reappraisal (e.g., Dillon et al., 2007) carries over and enhances cognitive control for a short period following the regulation event. Decreasing negative emotions through cognitive reappraisal, on the other hand, had no effect on subsequent Stroop performance or Stroop-related ERPs, suggesting that the consequences for cognitive control are not uniform across all forms of reappraisal.

That Stroop interference was reduced following instructions to increase negative emotions through reappraisal suggests an enhancement of cognitive control that extends for at least a brief period after the regulation event. The enhanced difference in the Stroop-related SP further bolsters this interpretation, since the SP is associated with recruitment of response-related control processes instantiated in the PFC, ACC, and extrastriate cortex (Lansbergen 
Decrease Block

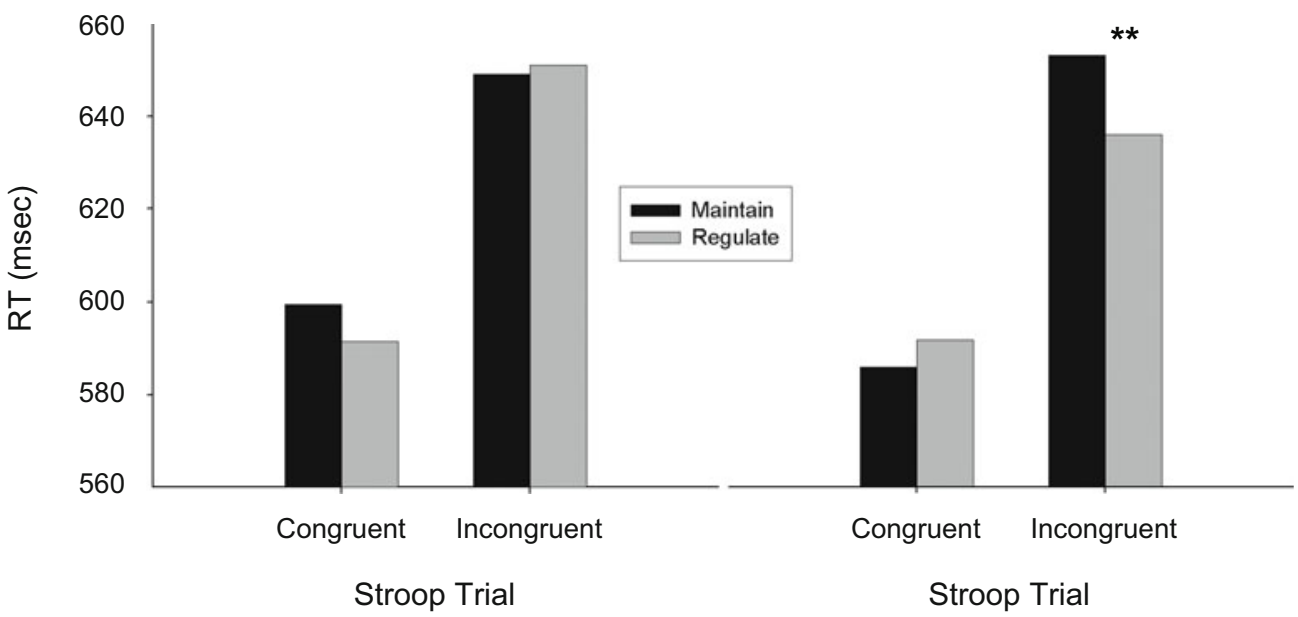

Figure 3. Response time (RT) data demonstrating the differential Stroop congruency effect in the decrease and increase blocks. ${ }^{* *} p=.01$.

et al., 2007; West, 2003; West et al., 2005). Although the SP showed modulation by increase instructions, the N450 did not. Research suggests that the N450 is related to early response-monitoring stages of conflict processing, whereas the SP indexes later response resolution and selection stages (Lansbergen et al., 2007; West, 2003; West et al., 2005). In the context of this research, the present results suggest that increasing negative emotions by cognitive reappraisal may specifically enhance later stages of subsequent conflict processing aimed at resolving response conflict and selecting appropriate responses, as indexed by the SP. Our finding of improved Stroop performance and associated SP modulation following increase instructions suggests that underlying processes that, in previous studies, had led to enhanced memory for emotional pictures (Dillon et al., 2007) carry over for a short time after the reappraisal period, with benefits for cognitive control over temporally proximal stimuli.

These findings stand in contrast to Deveney and Pizzagalli's (2008) results, however, which demonstrated reduced allocation of attention, as indexed by the P300, to emotional words following emotional enhancements through reappraisal. This discrepancy might simply be attributable to the differences between the emotion identification task used in their study and the Stroop task utilized here. That is, the availability of resources for processing emotional stimuli might be reduced following emotion regulation, for the simple reason that both tasks involve emotion processing. Another difference between our study and theirs is the timing of stimuli: Deveney and Pizzagalli employed a substantially longer picture presentation/regulation period $(10 \mathrm{sec}$, with $5 \mathrm{sec}$ of viewing and $5 \mathrm{sec}$ of regulation) than we did here ( $2 \mathrm{sec}$ of picture presentation and regulation combined, plus a variable, brief pre-Stroop interval), and it is possible that longer regulation periods drain resources for subsequent processing, whereas shorter regulation periods prime subsequent cognitive control. The consequences of the emo- tion regulation time course for cognitive control represent an exciting and important avenue for future research.

Whereas cognitive control seemed to be enhanced following instructions to increase a negative emotional response through reappraisal, there was no effect on Stroop performance or Stroop-related ERPs following decrease instructions, even in the face of successful downregulation (as indexed by both self-report and decreased LPP magnitude). On the face of it, this result is difficult to reconcile with the combination of our results in the increase condition and previous results suggesting that decrease instructions also involve enhanced engagement of cognitive processes, as reflected in increased attention to and elaboration of emotional stimuli (Dillon et al., 2007; Richards et al., 2003; Richards \& Gross, 2000). Notably, however, Dillon and colleagues found that decreasing one's emotional response does not have as strong an effect on memory for reappraised material as does increasing one's emotional response (Dillon et al., 2007).

There are also two additional possible explanations for the asymmetrical effects of increase and decrease instructions. First, it is possible that decreasing emotions via reappraisal requires less cognitive control than does increasing emotions. This makes some intuitive sense: Assuming that images provoke transient emotional responses, which quickly crest and then recede, efforts aimed at decreasing such an emotional response would enjoy the benefit of one's natural emotional momentum, whereas efforts to increase such an emotional response would be directed against the tide of this emotional flow. Work on conflict adaptation $^{4}$ (Botvinick, Braver, Barch, Carter, \& Cohen, 2001; Egner, 2007; Gratton, Coles, \& Donchin, 1992), demonstrating reduced congruence effects on trials that follow high-conflict trials, provides potentially converging insight. The proposed mechanism behind the conflict adaptation effect is that high-conflict trials engage an increase in cognitive control that carries over to the next trial, resulting in improved target selection and, therefore, 

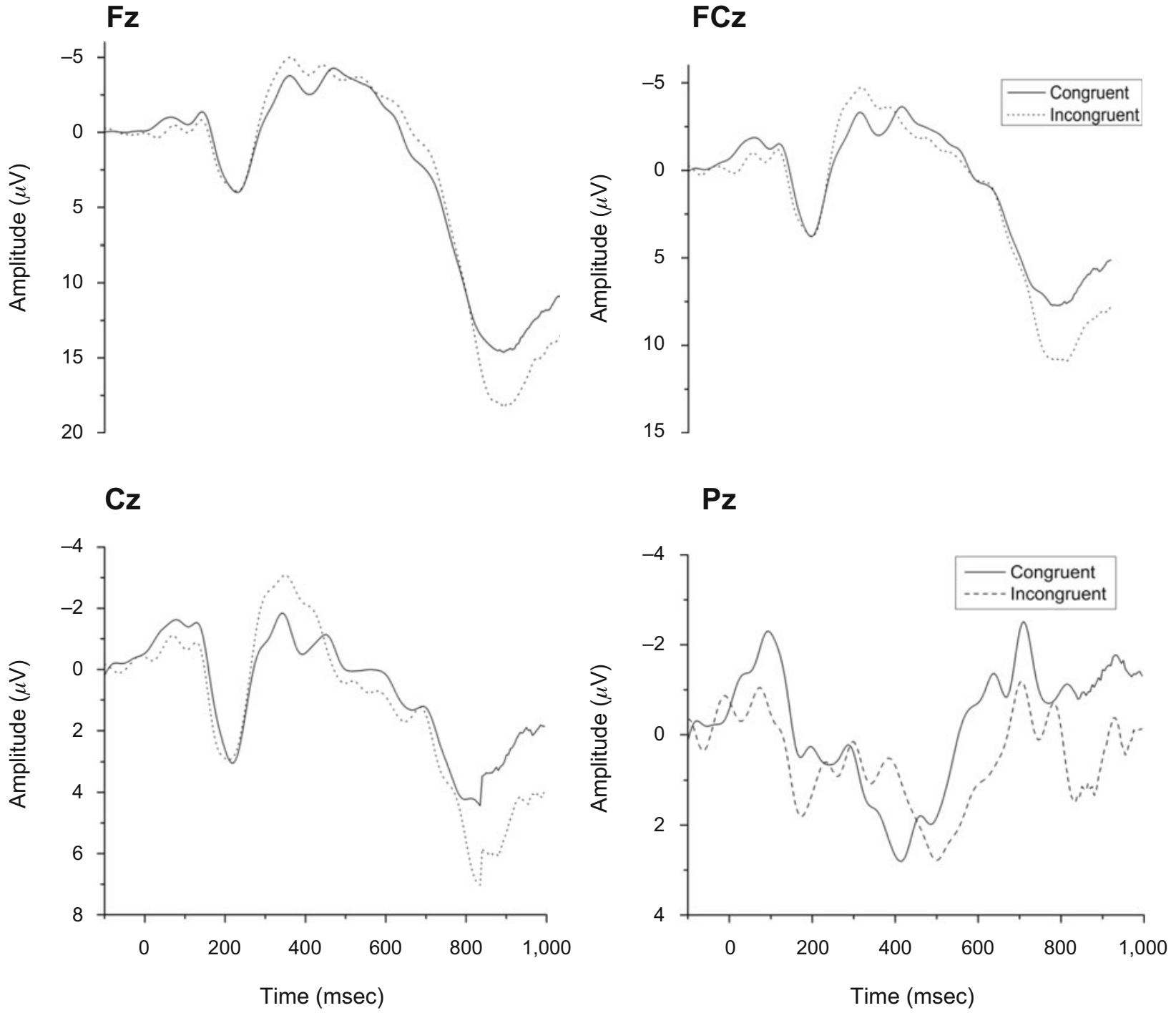

Figure 4. Stroop-locked ERPs at frontal (Fz), frontocentral (FCz), central (Cz), and parietal (Pz) locations for the increase block. Time point 0 represents Stroop onset.

reduced interference (Botvinick et al., 2001). In similar fashion, the extra effort involved in increasing negative emotions, relative to decreasing them, could trigger the enhanced recruitment of neural activity underpinning cognitive control on the subsequent Stroop trial. Two pieces of evidence support this interpretation: (1) Activity in the time range of the LPP - which is enhanced through reappraisal instructions to increase emotional response-has been linked to cognitive control and selective attention subserved by overlapping cortical structures (Larson et al., 2009; Nieuwenhuis et al., 2005), such as the frontoparietal network that plays a central role in monitoring the need for and implementing enhanced cognitive control (Egner et al., 2007; Liston et al., 2006; McClure et al., 2007; E. K. Miller \& Cohen, 2001; Ochsner \& Gross, 2005, 2007); and (2) the difference in Stroop SP as a function of congruence was enhanced following increase instructions and was negatively correlated with behavioral Stroop interfer- ence. Notably, in the present study, increase instructions seemed to have had a broad effect on the LPP across sites, whereas decrease instructions elicited LPP effects that were generally limited to the parietal lead; it is therefore possible that increase instructions had a more widespread effect on arousal and cognitive functions than did decrease instructions in the present context.

Second, although not mutually exclusive with the mechanism proposed above, it is also possible that differences between decrease and increase instructions were due to enhanced emotional arousal during increase instructions and reduced arousal during decrease instructions, consistent with studies suggesting that increased emotional arousal enhances cognitive functions such as attention and memory (e.g., LaBar \& Cabeza, 2006). A number of studies have suggested that negative emotional states focus processing resources for spatiotemporal information and enhance attentional control and spatial 
working memory (Gray, 2001; Gray, Braver, \& Raichle, 2002; Gray, Schaefer, Braver, \& Most, 2005; Jefferies, Smilek, Eich, \& Enns, 2008). Although these studies, as well as the present one, might demonstrate that negative emotional states enhance cognitive performance in some instances, future studies will be needed to clarify more specifically which cognitive processes are enhanced and which ones might be impaired. For example, it will be important to differentiate between the effects on cognitive processes of transient emotional states and the effects on cognition of trait-negative emotionality, as experienced by those diagnosed with mood and anxiety disorders (Hartston \& Swerdlow, 1999; Lemelin, Baruch, Vincent, Everett, \& Vincent, 1997). One possibility is that the pervasiveness and duration of negative affect determines the detrimental versus beneficial effects on cognitive functions (see Moser, Hajcak, \& Simons, 2005). This would be consistent with the possible role of trial duration in accounting for the apparent discrepancy between our results and those in Deveney and Pizzagalli (2008).

In light of previous studies showing that behavioral emotion regulation instructions such as suppression or enhancement of emotional expressions impair subsequent cognitive performance (Inzlicht \& Gutsell, 2007; Schmeichel, 2007; Shamosh \& Gray, 2007), it will be important for future research to continue to examine the differential effects of cognitive versus behavioral emotion regulation on cognition. Overall, our findings with reappraisal suggest that the depletion of cognitive resources associated with emotion regulation may arise specifically from behavioral regulation strategies, not from cognitive reappraisal. Our present finding that increasing negative emotions by reappraisal enhances subsequent cognitive control, whereas decreasing negative emotions by reappraisal neither enhances nor impairs subsequent cognitive control, is generally consistent with the view that reappraisal is not associated with the same cognitive costs as is behavioral emotion regulation (Richards \& Gross, 2000, 2006).

Although the enhanced LPP during increase instructions was followed by improved cognitive control on the Stroop task, its amplitude was not significantly correlated with subsequent Stroop performance or with Stroop-related ERPs. Although such a correlation would have lent further support to our claims, the lack of it does not preclude the possibility of a mechanistic relationship between the two. For example, such a relationship could have been obscured by the fact that ERPs generally reflect finer-grained processes, whereas behavior is an amalgam of different mechanisms, thus entailing an unwieldy degree of noise in this analysis. Indeed, the LPP is a relatively late ERP component that possibly reflects a number of cognitive and emotional processes (Hajcak \& Nieuwenhuis, 2006; Larson et al., 2009; Olofsson et al., 2008), and thus, it likely contains substantial noise in and of itself. Nevertheless, it is important to note that an enhanced LPP emerged from the same set of trials in which Stroop performance was enhanced, thus leaving open the possibility of a relationship between the two. Future studies should evaluate other cognitive tasks that may be more sensitive to relationships with the LPP.
One possible limitation of the present study is that we collected self-reported affect and effort ratings only at the conclusion of the experiment. Primarily, this was a manipulation check, and placing it at the end of the experiment ensured that the complexity of the task was kept to a minimum. Instead, we used the LPP as our primary index of reappraisal processes so as to protect against demand characteristics that can affect self-report measures in such tasks. Nevertheless, future researchers should consider recording other objective measures of affect and effort in order to elucidate further their respective contributions to reappraisal and their impact on subsequent cognitive processing.

In sum, cognitively increasing negative emotional responses to unpleasant pictures enhanced subsequent cognitive control, as evidenced by improved Stroop performance and modulation of its related neural measure (the SP), and elicited a larger LPP magnitude during reappraisal. The present findings suggest that cognitive control is an important mechanism for, and is subsequently affected by, reappraisal. They further suggest that such effects may be specific to the increasing of one's emotional response, since instructions to decrease one's negative emotional response failed to affect subsequent cognitive control. These results have implications for the broader construct of self-regulation (Muraven \& Baumeister, 2000) and suggest that not all forms of self-regulation are created equal; some forms of self-regulation seem to deplete information-processing resources, whereas others might enhance them.

\section{AUTHOR NOTE}

Portions of this article were presented at the 48th Annual Meeting of the Society for Psychophysiological Research, Austin, TX, October 2008. This research was supported in part by a National Institute of Mental Health Predoctoral Fellowship (MH077388) awarded to the first author. We thank Greg Hajcak and Dan Dillon for their extremely helpful comments on a previous draft of the manuscript. J.S.M. is now in the Department of Psychology at Michigan State University. Correspondence concerning this article should be addressed to J. S. Moser, Department of Psychology, Michigan State University, East Lansing, MI 48824 (e-mail: jmoser@msu.edu).

\section{REFERENCES}

Bonanno, G. A., Papa, A., Lalande, K., Westphal, M., \& CoifMAN, K. (2004). The importance of being flexible: The ability to both enhance and suppress emotional expression predicts long-term adjustment. Psychological Science, 15, 482-487.

Botvinick, M. M., Braver, T. S., Barch, D. M., Carter, C. S., \& Cohen, J. D. (2001). Conflict monitoring and cognitive control. Psychological Review, 108, 624-652.

Cook, E. W., III (1999). VPM reference manual. Birmingham, AL: Author.

CoOK, E. W., III, \& Miller, G. A. (1992). Digital filtering: Background and tutorial for psychophysiologists. Psychophysiology, 29, 350-367.

Deveney, C. M., \& Pizzagalli, D. A. (2008). The cognitive consequences of emotion regulation: An ERP investigation. Psychophysiology, 45, 435-444.

Dillon, D. G., Ritchey, M., Johnson, B. D., \& LaBar, K. S. (2007). Dissociable effects of conscious emotion regulation strategies on explicit and implicit memory. Emotion, 7, 354-365.

Donchin, E. (1981). Surprise! ... Surprise? Psychophysiology, 18, 493513.

EGNER, T. (2007). Congruency sequence effects and cognitive control. Cognitive, Affective, \& Behavioral Neuroscience, 7, 380-390. 
Egner, T., Delano, M., \& Hirsch, J. (2007). Separate conflict-specific cognitive control mechanisms in the human brain. NeuroImage, $\mathbf{3 5}$ 940-948.

Ellis, H. C., \& Ashbrook, P. W. (1988). Resource allocation model of the effects of depressed mood states on memory. In K. Feidler \& J. Forgas (Eds.), Affect, cognition and social behavior (pp. 25-43). Toronto: Hogrefe.

Gehring, W. J., Goss, B., Coles, M. G. H., Meyer, D. E., \& DonCHIN, E. (1993). A neural system for error detection and compensation. Psychological Science, 4, 385-390.

Gratton, G., Coles, M. G. H., \& Donchin, E. (1983). A new method for off-line removal of ocular artifact. Electroencephalography \& Clinical Neurophysiology, 55, 468-484.

Gratton, G., Coles, M. G. H., \& Donchin, E. (1992). Optimizing the use of information: Strategic control of activation of responses. Journal of Experimental Psychology: General, 121, 480-506.

GRAY, J. R. (2001). Emotion modulation of cognitive control: Approachwithdrawal states double-dissociate spatial from verbal two-back task performance. Journal of Experimental Psychology: General, 130, 436-452.

Gray, J. R., Braver, T. S., \& Raichle, M. E. (2002). Integration of emotion and cognition in the lateral prefrontal cortex. Proceedings of the National Academy of Sciences, 99, 4115-4120.

Gray, J. R., Schaefer, A., Braver, T. S., \& Most, S. B. (2005). Affect and the resolution of cognitive control dilemmas. In L. FeldmanBarrett, P. Niedenthal, \& P. Winkielman (Eds.), Emotion: Conscious and unconscious (pp. 67-94). New York: Guilford.

Gross, J. J. (ED.) (2007). Handbook of emotion regulation. New York: Guilford.

Gross, J. J., \& Thompson, R. A. (2007). Emotion regulation: Conceptual foundations. In J. J. Gross (Ed.), Handbook of emotion regulation (pp. 3-26). New York: Guilford.

HAJCAK, G., \& NiEUWENHUIS, S. (2006). Reappraisal modulates the electrocortical response to unpleasant pictures. Cognitive, Affective, \& Behavioral Neuroscience, 6, 291-297.

Hartston, H. J., \& Swerdlow, N. R. (1999). Visuospatial priming and Stroop performance in patients with obsessive compulsive disorder. Neuropsychology, 13, 447-457.

InZlicht, M., \& Gutsell, J. N. (2007). Running on empty: Neural signals for self-control failure. Psychological Science, 18, 933-937.

Jackson, D. C., Malmstadt, J. R., Larson, C. L., \& Davidson, R. J. (2000). Suppression and enhancement of emotional responses to unpleasant pictures. Psychophysiology, 37, 515-522.

Jefferies, L. N., Smilek, D., Eich, E., \& EnNs, J. T. (2008). Emotional valence and arousal interact in attentional control. Psychological Science, 19, 290-295.

Kliegel, M., Horn, A. B., \& Zimmer, H. (2003). Emotional aftereffects on the $\mathrm{P} 3$ component of the event-related brain potential. International Journal of Psychophysiology, 38, 129-137.

Krompinger, J. W., Moser, J. S., \& Simons, R. F. (2008). Modulations of the electrophysiological response to pleasant stimuli by cognitive reappraisal. Emotion, 8, 132-137. doi:10.1037/1528-3542.8 .1 .132

LABAR, K. S., \& CABEZA, R. (2006). Cognitive neuroscience of emotional memory. Nature Reviews Neuroscience, 7, 54-64.

LANG, P. J., Bradley, M. M., \& Cuthbert, B. N. (1999). International Affective Picture System: Instruction manual and affective ratings (Tech. Rep. A-4). Gainesville: University of Florida, Center for Research in Psychophysiology.

Lansbergen, M. M., van Hell, E., \& Kenemans, J. L. (2007). Impulsivity and conflict in the Stroop task: An ERP study. Journal of Psychophysiology, 21, 33-50.

Larson, M. J., Kaufman, D. A. S., \& Perlstein, W. M. (2009). Neural time course of conflict adaptation effects on the Stroop task. Neuropsychologia, 47, 663-670.

Lemelin, S., Baruch, P., Vincent, A., Everett, J., \& Vincent, P. (1997). Distractibility and processing resource deficit in major depression: Evidence for two deficient attentional processing models. Journal of Nervous \& Mental Disease, 185, 542-548.

Liston, C., Matalon, S., Hare, T. A., Davidson, M. C., \& Casey, B. J. (2006). Anterior cingulate and posterior parietal cortices are sensitive to dissociable forms of conflict in a task-switching paradigm. Neuron, 50, 643-653.

McClure, S. M., Botvinick, M. M., Yeung, N., Greene, J. D., \& Cohen, J. D. (2007). Conflict monitoring in cognition-emotion competition. In J. J. Gross (Ed.), Handbook of emotion regulation (pp. 204-226). New York: Guilford.

Miller, E. K., \& COHEN, J. D. (2001). An integrative theory of prefrontal cortex. Annual Review of Neuroscience, 24, 167-202.

Miller, G. A., Gratton, G., \& Yee, C. M. (1988). Generalized implementation of an eye movement correction procedure. Psychophysiology, 25, 241-243.

Monsell, S. (2003). Task switching. Trends in Cognitive Sciences, 7, 134-140.

Moser, J. S., Hajcak, G., Bukay, E., \& Simons, R. F. (2006). Intentional modulation of emotional responding to unpleasant pictures: An ERP study. Psychophysiology, 43, 292-296. doi:10.1111/j.1469 -8986.2006.00402.X

Moser, J. S., HajcaK, G., \& Simons, R. F. (2005). The effects of fear on performance monitoring and attentional allocation. Psychophysiology, 42, 261-268. doi:10.1111/j.1469-8986.2005.00290.x

Moser, J. S., Krompinger, J. W., Dietz, J., \& Simons, R. F. (2009). Electrophysiological correlates of decreasing and increasing emotional responses to unpleasant pictures. Psychophysiology, 46, 17-27. doi:10.1111/j.1469-8986.2008.00721.x

Muraven, M., \& Baumeister, R. F. (2000). Self-regulation and depletion of limited resources: Does self-control resemble a muscle? Psychological Bulletin, 126, 247-259.

Nieuwenhuis, S., Aston-Jones, G., \& Cohen, J. D. (2005). Decision making, the P3, and the locus coeruleus norepinephrine system. Psychological Bulletin, 131, 510-532.

OCHSNER, K. N., \& Gross, J. J. (2005). The cognitive control of emotion. Trends in Cognitive Sciences, 9, 242-249.

OCHSNER, K. N., \& GRoss, J. J. (2007). The neural architecture of emotion regulation. In J. J. Gross (Ed.), Handbook of emotion regulation (pp. 87-109). New York: Guilford.

Ochsner, K. N., Ray, R. D., Cooper, J. C., Robertson, E. R., Chorpa, S., Gabrieli, J. D. E., \& Gross, J. J. (2004). For better or for worse: Neural systems supporting the cognitive down- and upregulation of negative emotion. NeuroImage, 23, 483-499.

Olofsson, J. K., Nordin, S., Sequeira, H., \& Polich, J. (2008). Affective picture processing: An integrative review of ERP findings. Biological Psychology, 77, 247-265.

Richards, J. M., ButLER, E. A., \& Gross, J. J. (2003). Emotion regulation in romantic relationships: The cognitive consequences of concealing feelings. Journal of Social \& Personal Relationships, 20, 599-620.

Richards, J. M., \& Gross, J. J. (1999). Composure at any cost? The cognitive consequences of emotion suppression. Personality \& Social Psychology Bulletin, 25, 1033-1044.

Richards, J. M., \& Gross, J. J. (2000). Emotion regulation and memory: The cognitive costs of keeping one's cool. Personality Processes \& Individual Differences, 79, 410-424.

Richards, J. M., \& Gross, J. J. (2006). Personality and emotional memory: How regulating emotion impairs memory for emotional events. Journal of Research in Personality, 40, 631-651.

Schmeichel, B. J. (2007). Attention control, memory updating, and emotion regulation temporarily reduce the capacity for executive control. Journal of Experimental Psychology: General, 136, 241-255.

Shamosh, N. A., \& GRAY, J. R. (2007). The relation between fluid intelligence and self-regulatory depletion. Cognition \& Emotion, 21, 1833-1843.

WEST, R. (2003). Neural correlates of cognitive control and conflict detection in the Stroop and digit-location tasks. Neuropsychologia, 41, 1122-1135.

West, R., JakubeK, K., Wymbs, N., Perry, M., \& Moore, K. (2005). Neural correlates of conflict processing. Experimental Brain Research, 167, 38-48.

WiNDES, J. D. (1968). Reaction time for numerical coding and naming of numerals. Journal of Experimental Psychology, 78, 318-322.

Zelazo, P. D., \& Cunningham, W. (2007). Executive function: Mechanisms underlying emotion regulation. In J. J. Gross (Ed.), Handbook of emotion regulation (pp. 135-158). New York: Guilford. 


\section{NOTES}

1. The numbers of the IAPS pictures used were the following: 1050, 1052, 1090, 1110, 1113, 1114, 1120, 1200, 1201, 1205, 1220, 1300, $1301,1321,1525,1930,3000,3010,3030,3051,3053,3060,3061$, 3062, 3063, 3064, 3069, 3100, 3102, 3110, 3130, 3170, 3266, 6230, $6242,6243,6244,6250,6260,6313,6315,6350,6360,6540,6550$, $6560,6570,6821,9410,9800$.

2. Verbatim instructions for the decrease trials were as follows:

When you see the word "DECREASE", you should think about the following picture in such a way that you feel your negative emotions less strongly. For example, you could view the picture as a detached, third-person who is not present at the scene pictured. If you were viewing an image of a sick person you could view this person from a detached, clinical perspective like someone not personally connected in any way to the individual. You can think of the picture as being fake or from a movie. You could also imagine that the pictured event gets better. If you were viewing an image of a sick person you could imagine that the person is not experiencing any pain and will recover quickly. The only thing I ask that you do not do is to simply think about something unrelated to the scene. For example, do not simply "think happy thoughts" in order to de- crease your negative emotions. Just try to reappraise the context of the scene as I just described.

3. Verbatim instructions for the increase trials were as follows:

When you see the word "INCREASE", you should think about the following picture in such a way that you feel your negative emotions more strongly. For example, if you were viewing an image of a sick person you could imagine that you or a loved one are this individual, or that you are observing that individual suffering in their hospital bed. You could also imagine that the pictured event gets worse. If you were viewing an image of a sick person you could imagine that the person is in great pain and is unlikely to ever recover. The only thing that I ask that you do not do is to simply think about something unrelated to the scene. For example, do not simply think of an unrelated negative situation in order to make your negative emotions more prominent. Just try to reappraise the context of the scene as I just described.

4. We thank Amishi Jha for this insight.

(Manuscript received August 16, 2008;

revision accepted for publication November 14, 2009.) 